\title{
Anesthesia and Anesthetist: People's Perception and Reality
}

HK Mahajan

How to cite this article: Mahajan HK. Anesthesia and Anesthetist: People's Perception and Reality. Int J Recent Surg Med Sci 2017;3(2):73-74.

\section{Source of support: Nil}

\section{Conflict of interest: None}

The success of anesthesia in common perception is based on the anesthetist's ability to induce a smooth sleep, provide constant anesthesia during surgery, and a guarantee of smooth wake-up after the procedure. It is also expected that pain will not be perceived while the patient is under the influence of anesthesia. But, people have concerns about awakening from anesthesia and have deep-rooted fear that they either may never get up from the surgical table or anesthesia may wean off during the surgical procedure itself and they may get up in between.

Despite the common fears, most people think anesthesia to be a relatively safe event, and administration of anesthesia to be a relatively simple procedure which even a nonmedical staff or a surgeon who is untrained in the practice of anesthesia can easily manage. But in reality, anesthesia is one of the most risk-prone specialty in medical practice. A patient once anesthetized is completely in the hands of the anesthetist and a slight error of judgment on the part of the anesthesiologist can have serious consequences for the patients. Furthermore, the fact that all anesthesiologists around the world are medical practitioners and have to receive substantial amount of training in the specialty before being authorized to do independent practice is unknown to most of the people.

The reasons for development of such perceptions regarding anesthesia and its practitioners may lie in the history of the specialty itself.

The first demonstration of anesthesia on a patient during surgery was done more than 170 years ago in 1846 by WTG Morton who was a dentist by profession.

Chief

Department of Anesthesia and Intensive Care, Indian Spinal Injuries Centre, New Delhi, India

Corresponding Author: HK Mahajan, Chief, Department of Anesthesia and Intensive Care, Indian Spinal Injuries Centre New Delhi, India, e-mail: hod.anaesthesia@isiconline.org
He used inhaled ether to give anesthesia to his patient Mr Gilbert Abbot for removal of a neck tumor. Since then, for about 100 years, only three anesthetic agents - ether, chloroform, and nitrous oxide, remained in common use. Also, the doctors who practiced anesthesia during these early years were either junior surgeons from the surgical team or physicians. None of them were full-time practicing anesthesiologists. Here reference should be made of John Snow who was the most renowned physician and epidemiologist of the 19th century. He is credited as the first full-time anesthesiologist and he did a lot of work for the development of anesthesia and invented many equipments for safe and effective delivery of anesthesia.

The use of ether and chloroform in the early years of this specialty was responsible for all the common problems which most people still associate with anesthesia. Although these two provided good analgesia and were relatively safe agents to use, they had a very stormy induction and recovery and most people experienced a lot of nausea and vomiting and had unpleasant experiences during recovery. Furthermore, most of the hospitals in developing countries like India were not fully equipped for administration of anesthesia and for proper monitoring of patients during the process. As a result, quite often the anesthesiologists had to use minimal amount of anesthetic drugs in order to maintain patient safety. This resulted in inadequate anesthesia delivery and feelings of awareness and pain in the patients during anesthesia procedure, and hence the development of perception of weaning of anesthesia during the surgery and the fear associated with it.

Since the 1920s and 1930s, the practice of anesthesia slowly began to change. From being a subsidiary branch of surgery, it started to evolve as a full-fledged independent specialty. It developed its own training program and certification, which was at par with any medical specialty.

The anesthetic drugs also changed from unpleasant anesthesia of ether and chloroform to thiopentone and halothane and now to propofol, sevoflurane, remifentanil, and atracurium. These newer drugs have very fast induction and recovery and smooth course during surgery. Also, patient wakes up with a clear consciousness and usually does not have postprocedural complaints like nausea, vomiting, pain etc. Many other drugs are also available, which have reduced the adverse effects to a minimum. 
The level of sedation which is used for surgeries nowadays has also changed. Deeper levels of anesthesia are being used nowadays. Sicker patients and long duration complicated surgeries which were considered unfit for anesthesia earlier are now being routinely done. This has become possible because of better availability of life support equipment, such as ventilators, safer anesthesia workstations, electronic monitors which provide the anesthesiologists beat to beat data of patient's hemodynamic status and better equipped intensive care units (ICUs) for shifting of postoperative patients. Anesthesiologists nowadays receive training in anesthesia subspecialties also, such as cardiac anesthesia, neuroanesthesia, pain medicine, and ICU care.
Similarly, in case of regional anesthesia procedures, lots of improvements have been made. Needles used now for giving the blocks are thinner and are made of disposable material. Drugs which are used to achieve regional anesthesia are more effective and safe than ever. Ultrasound machines are also getting used in all good centers around the world, to achieve pinpoint anesthesia and better-quality blocks.

All these advances in anesthesia have changed the specialty very much, and despite its inherent risks have made it quite safe. Perioperative events like pain, awareness, nausea, etc., as well as the extremely rare event of patient not awakening from anesthesia are now stories of the past. 